\title{
Electrical Conductivity Extracted From Optical Characterization of Polysilicon Films
}

\author{
Boubekeur Birouk ${ }^{1,2}$, Jean-Pierre Raskin ${ }^{1}$ \\ ${ }^{1}$ ICTEAM/ Université catholique de Louvain \\ Maxwell building, Place du Levant, 3, 1348Louvain-la-Neuve, Belgium \\ boubekeur.birouk@uclouvain.be; jean-pierre.raskin@uclouvain.be \\ ${ }^{2}$ FST/Université de Jijel \\ Ouled Aissa Zone, 18000Jijel, Algérie \\ bbirouk@gmx.com
}

\section{Extended Abstract}

The purpose of this work is to investigate the optical properties of polycrystalline silicon layers by means of spectroscopic Ellipsometry and other techniques to sustain the analysis (SEM, AFM and Hall effect).The studied MOS structures are composed of c-Si substrate(p-type, $\mathrm{Cz}<100\rangle$ oriented), silicon oxide layer $(100 \mathrm{~nm})$ and polysilicon film $\left(175 \mathrm{~nm}\right.$ deposited by LPCVD at $625^{\circ} \mathrm{C}$ ) with several doping levels(from $2 \times 10^{19}$ to $\left.3 \times 10^{20} \mathrm{~cm}^{-3}\right)$.A five-layers structure model has been applied, including in addition to MOS structure, a native oxide layer and an effective medium approximation (EMA) roughness layer based on linear growth of air medium. A Cauchy layer model was used to compute the optical parameters (for 400-900 $\mathrm{nm}$ wavelength range). The fitting sessions lead to good results as the theoretical and experimental ellipsometric angles curves superposed to each other. The total error was lowered to its minimum during fitting process, by reducing the partial errors on which one can act, like fixed values, misalignment of the angle of incidence [1], bad software convergence, specific measuring error,; and so on.

Firstly, the SEM images correlated with AFM ones, of the doped or undoped deposited polysilicon, show sugar loaf shaped surface crystallites, like it appears in reference [2]. The Ellipsometry study showed that the polySi layer roughness has undergone a growth under the effect of the phosphorus thermal diffusion. It increases from $22.2 \AA$ to a value between 23 and $58 \AA$, depending on the doping level, which agrees with SEM/AFM characterizations and what has been published elsewhere $[3,4]$, in case of the temperature deposition of $625^{\circ} \mathrm{C}$.

Secondly, one noticed that ellipsometric angles curves were regularly shifted towards low wavelengths when the electrical conductivity increases and the wavelengths gap between the extrema angles, for the same curve, was monotonically decreasing in the same situation. Besides, Psi angle maxima and minima were growing till the doping level reaches a limit (when approaching the phosphorus solubility limit), then started to diminish. On the other hand, the Mean Square Error (MSE) [5,6], when determined between experimental curves, after and before phosphorus diffusion, linearly increases with conductivity enhancement. We took advantage of these curves properties for determining of the electrical conductivity (and resistivity) of the deposited PolySi films, by means of simple relationships between the latter, on the one hand, and the ellipsometric angles extrema and associated wavelengths, on the other hand. Even if this method is not a straightforward manner to extract the conductivity, it remains a good way to avoid electrical contacts on samples, mainly in case of small areas. The evolution of Delta and Psi curves (shift and extrema values variation) can be related to two main influent sources. In the first place, the evolution of the free electrons concentration modifies the complex refractive index in accordance to the Drude theory (correlation between SE and HE measurements). Secondly, the polysilicon layer crystallinity and roughness increase with the doping level, following the thermal budget during the diffusion process.

Thirdly, the characterizations confirmed that the refractive index is reduced by the doping process, and that it is all lower as the phosphorus doping level is higher. In addition, we noticed that refractive indices evolution are, in all cases, in agreement with model that has been published in[7], in other words their values decrease on Vis-NIR domain, in accordance with a seven-terms polynomial function $\mathrm{n}^{2}=\mathrm{f}\left(\lambda^{2}\right)$, with alternate signs. Furthermore, the seven parameters fall on Gaussian curves and are very depending on the doping and the polysilicon layer thickness.

\section{References}

[1] H. Reisinger, "Minimization of errors in ellipsometric measurements," Solid-State Electronics, vol. 35, no. 3, pp. 333-344, 1992. 
[2] L. Rolland, C. Vallée, M.-C. Peignon, and C. Cardinaud; "Roughness and chemistry of silicon and polysilicon surfaces etched in high-density plasma: XPS, AFM and Ellipsometry analysis," Applied Surface Science, 164, pp. $147-155,2000$.

[3] H. Günther, "Optical Properties of Polycrystalline Silicon Films," Springer Series in Solid-State Sciences, vol. 57, pp. 156-169, 1985.

[4] P. Petrik, L. P. Biro, M. Fried, T. Lohner, R. Berger, C. Schneider, J. Gyulai, and H. Ryssel, "Comparative study of surface roughness measured on polysilicon using spectroscopic ellipsometry and atomic force microscopy," Thin Solid Films, vol. 315, pp. 186-191, 1998.

[5] B. Gruska, "Ellipsometric analysis of polysilicon layers," Thin Solid Films, vol. 364, pp. 138-143, 2000.

[6] M. Modreanu, M. Gartner, C. Cobianu, B. O’Looney, and F. Murph, "Optical properties of silicon thin films related to LPCVD growth condition," Thin Solid Films, vol. 450, pp. 105-110, 2004.

[7] B. Birouk and D.Madi, "Thermal oxidation effect on structural and optical properties of heavily doped phosphorus polycrystalline silicon films," Appl. Phys. A, vol. 104, no. 2, pp. 739-748, 2011. 